\title{
Vancomycin Resistant Enterococci in Makkah Workers
}

\section{Hussam Aldeen Hassan ${ }^{1 *}$, Abdalla Osman Abdalla Ahmed ${ }^{2}$ and Bashir Sirag $^{3}$}

${ }^{1}$ Medical Laboratory Specialist in Zaher Gadeeb Al-Baan Polyclinic, Makkah, Saudi Arabia

${ }^{2}$ Associate Professor, Department of Medical Microbiology, Faculty of Medicine, Umm Al-Qura University, Saudi Arabia

${ }^{3}$ Microbiology Department, Faculty of Medicine, Umm Al-Qura University, Saudi Arabia

*Corresponding Author: Hussam Aldeen Hassan, Medical Laboratory Specialist in

Zaher Gadeeb Al-Baan Polyclinic, Makkah, Saudi Arabia.
Received: August 17, 2020

Published: October 15, 2020

(C) All rights are reserved by Hussam

Aldeen Hassan., et al

\section{Abstract}

Aim: The aim of this study is to determine the vancomycin susceptibility in intestinal isolates of Enterococci isolated from healthy workers in Makkah.

Methods: disk diffusion technique was used in this study to determine the antimicrobial susceptibility for Penicillin, Ampicillin, Vancomycin and Linezolid. PCR testing for major resistant genes was also used.

Results: No resistant isolate was detected by disk diffusion technique. However, eight samples were PCR positive for Van B, two isolates were positive for Van C and only one isolate was positive VanC2/C3.

Conclusions: all isolates were sensitive by disc diffusion method, and Van genes were detected by PCR in few numbers of isolates.

Keywords: Enterococci; Streptococci; Ampicillin; Penicillin

\section{Introduction}

Formerly enterococci categorized in genus streptococci (group D streptococci or "streptococci of fecal origin". which has the ability to resist chemical and physical agents, molecular methods help to separate this group to separate group named Enterococci [1]. First species were separated to new taxonomy Enterococcus faecalis and Enterococcus faecium. Enterococcus faecalis may appear hemolytic in blood agar if agar prepared from rabbit or horse, nonhaemolytic if agar prepared from sheep blood agar. Uses of molecular detection such as PCR for detection of enterococcal infection it's more valuable than culture. Because this method is sensitive and detection occur at gene level [2].

Enterococci is one of the gastrointestinal flora of human. Pathogenicity occurs due to some alteration e. g. prolonged antibiotic treatments, severe underlying diseases and impaired immune system. If enterococci become pathogen infect urinary tract and intraabdominal infections, bacteremia, and infective endocarditis [3]. Presence of enterococci important sign of enterococcal infection in sterile specimens such a blood, but important to isolation and identification in non-sterile specimen culture [2]. In certain condition may cause some infection in a human thought transition of enterococci from habitat to play a major role change organism from commensal to infected organism [1]. Resistant to antimicrobial agents consider one of the characters of enterococci. E. faecalis and E. faecium concerned with human infection $[4,5]$.

Resistance to vancomycin due to presence of six genes which called Van genes (VanA, VanB, VanC, VanD, VanE, and VanG), most common are VanA and Van B [6]. 
For the first time, the enterococci resistance of Vancomycin was detected in 1986 [7]. Over the past 20 years, VRE has been considered one major of the leading causes of bacteremia of patients in hospitals, Enterococcus faecium is one of the strains that have managed to settle and adapt themselves in the hospital environment, Infection control procedures have failed to eliminate bacteremia occur by VRE moreover in some countries their rates have increased [8]. One of the most serious things in enterococci that it has the enormous ability to acquire antibiotic resistance to its genome from other sources as well as its original intrinsic resistant to antimicrobial agents. When we say that enterococci are resistant to Vancomycin this means they are resistant to a wide spectrum of antibiotics such as beta-lactam group. Recently, the World Health Organization (WHO) has classified E. faecium among the highly capable microbes his ability to develop new antibiotic resistance strategies [9].

VanA with high-level vancomycin resistance and multidrug resistance, it is present in countries that lack an infection control system. It has been discovered in healthy humans, animals and patients [10]. VanA transposon Tn1546, mostly responsible from acquired vancomycin resistant in Enterococcus faecium this transposon carried in plasmid [11].

\section{Materials and Methods}

This cross-sectional laboratory base study was carried out in Zaher Gadeeb Al-Baan Polyclinic, Asia polyclinic and Al Nour Specialized Hospital in Makkah Holy City, Saudi Arabia in period between 2015 to 2017.

710 stool specimens were collected from a healthy non-Saudi workers population performing their routine medical check.

Samples were directly cultured on MacConkey agar plates (MacConkey agar without salt $\mathrm{No}_{2}$, Oxoid).

After identification of enterococci, the sensitivity test was then performed. Antimicrobial susceptibility for Penicillin, ampicillin, vancomycin, and linezolid was be performed using disk diffusion method on Mueller Hinton agar.

Results were interpreted according to Clinical and Laboratory Standards Institute guidelines (CLSI guideline "Performance Standards for Antimicrobial Susceptibility Testing Document number M100-S24 2017).
Samples were harvested and stored in freezing media $(10 \%$ glycerol in nutrient broth) at $-20^{\circ} \mathrm{C}$.

Bacterial cells were suspended in Tris-EDTA buffer and $0.1 \mathrm{~mm}$ glass beads was added to the harvested cells and subjected to 2 rounds of beads-beating in Mini Bead-beater 16 (Biospec Inc, USA) followed by cooling in ice. DNA was then purified using phenolchloroform. Extracted DNA was quantified using Qubit Fluorometer (Invitrogen, USA).

\section{PCR and gel electrophoresis}

- $\quad$ PCR has been done for 120 enterococci isolates obtained from 710 stool samples.

- $\quad$ PCR was performed using AmpliTaq Gold 360 master mix from Applied Biosystems CA USA. $0.5 \mu \mathrm{l}$ of each primer was added to the working concentration master mix with $5 \mu$ l of each sample.

- Four Primers were used in PCR that targets VanA, VanB, VanC1, and Van C2/C3 genes [12].

- $\quad$ PCR reaction condition for Van A, Van B, Van C1 and Van C2/ $\mathrm{C} 3$ genes consist of Initial denaturation at $95^{\circ} \mathrm{C}$ for 10 mins; followed by 40 cycle of Denaturation at $95^{\circ} \mathrm{C}$ for $15 \mathrm{sec}$; annealing at $45.9^{\circ} \mathrm{C}$ for Van A gene, at $51.4^{\circ} \mathrm{C}$ for Van $\mathrm{B}$ gene, at $41.9^{\circ} \mathrm{C}$ for Van $\mathrm{C} 1 / \mathrm{C} 2 / \mathrm{C} 3$ genes respectively for $15 \mathrm{sec}$; followed by extension at $72^{\circ} \mathrm{C}$ for $30 \mathrm{sec}$, Final extension step was applied at $72^{\circ} \mathrm{C}$ for 5 mins.

- PCR products were analyzed on 1\% agarose gels in Tris/ Borate/EDTA buffer for 30 to 60 minutes and visualized using gel documentation system. Agarose gels were stained by ethidium bromide.

\section{Results}

One hundred and twenty Enterococci were identified by colonial morphology and direct Gram stain (Small fine red -magenta colour colonies). Showing Gram- positive cocci presumptively identified as enterococci. No VRE detect in healthy people. PCR done for 120 sample. All Van A were negative. VanB 8 samples were positive (5, $31,32,46,65,72,85$ and 98). Van C show 2 positive samples 6 and 86. Van $\mathrm{C} 2 / \mathrm{C} 3$ show only one sample positive.

\section{Discussion}

After examination of stool samples of the healthy workers, about 120 Enterococci isolated. None of the healthy workers were 
found to be colonized by VRE, although they are coming from different countries. This is suggesting of no prevalence of VRE in this community. As some previous study indicated VRE not found or rarely isolated from healthy $[13,14]$. This what was found in this collection of isolates. Normally VRE is more expected in hospital environments. We proofed that healthy workers are not harboring VRE and therefore they are not potential source of this dangerous phenotype.

In this study VanA gene mostly negative. VanA predominantly detected in the hospital setting [15].

VanB gene mostly negative but few isolates give positive results. There is no consistency between disc diffusion and PCR result because these genes has inducible resistant to vancomycin and may lead to inconsistent results between Disk diffusion methods and vanB detection by PCR [16]. In Some previous study VanB positive. Enterococcus faecium from rectal appear susceptible to vancomycin also VanB positive from clinical setting be more accessible to become resistant to Vancomycin [17].

Van C1 detected in 2 samples and van C2/C3 detected in only one sample. Van C1 and Van C2 genes associated with Enterococcus gallinarum and Enterococcus casseliflavus both are characteristic by low level resistant to vancomycin $[10,18,19]$.

\section{Conclusion}

According to the results obtained from PCR and gel electrophoresis, it seems that disk diffusion method must be used as a screening method and must be confirmed by another method especially in serious infections or in patients were infected by VRE in the past.

\section{Bibliography}

1. Lebreton F., et al. "Enterococcus Diversity, Origins in Nature, and Gut Colonization". In: GILMORE, M. S., CLEWELL, D. B., IKE, Y. and SHANKAR, N. (eds.) Enterococci: From Commensals to Leading Causes of Drug Resistant Infection. Boston (2014).

2. Teixeira LM., et al. "Enterococcus. Manual of Clinical Microbiology, 10th Edition". American Society of Microbiology (2011).

3. Crouzet L., et al. "Lactobacillus paracasei CNCM I-3689 reduces vancomycin-resistant Enterococcus persistence and promotes Bacteroidetes resilience in the gut following antibiotic challenge". Scientific Report 8 (2018): 5098.
4. Cheng M., et al. "The Bacteriophage EF-P29 Efficiently Protects against Lethal Vancomycin-Resistant Enterococcus faecalis and Alleviates Gut Microbiota Imbalance in a Murine Bacteremia Model". Frontiers in Microbiology 8 (2017): 837.

5. Summers NA., et al. "Multi-drug-resistant Enterococcus faecium bacteraemia in a liver transplant recipient". JMM Case Report 6 (2019): e005172.

6. Al-Ahdal MN., et al. "Characterization of Enterococcus faecium isolates and first report of vanB phenotype-vanA genotype incongruence in the Middle East". European Journal of Clinical Microbiology and Infectious Diseases 31 (2012): 3223-3229.

7. Benamu E and Deresinski S. "Vancomycin-resistant enterococcus infection in the hematopoietic stem cell transplant recipient: an overview of epidemiology, management, and prevention". F1000Research 7 (2018): 3.

8. Gouliouris T., et al. "Duration of exposure to multiple antibiotics is associated with increased risk of VRE bacteraemia: a nested case-control study". Journal of Antimicrobial Chemotherapy 73 (2018): 1692-1699.

9. Abdelkhalek A., et al. "Repurposing ebselen for decolonization of vancomycin-resistant enterococci (VRE)". PLoS One 13 (2018): e0199710.

10. Cha JO., et al. "Diversity of Tn1546 in vanA-positive Enterococcus faecium clinical isolates with VanA, VanB, and VanD phenotypes and susceptibility to vancomycin". Journal of Applied Microbiology 115 (2013): 969-976.

11. Oravcova V., et al. "Wild corvid birds colonized with vancomycin-resistant Enterococcus faecium of human origin harbor epidemic vanA plasmids". Environment International 118 (2018): 125-133.

12. Dutka-Malen S., et al. "Detection of glycopeptide resistance genotypes and identification to the species level of clinically relevant enterococci by PCR". Journal of Clinical Microbiology 33 (1995): 1434.

13. Gaca A O and GILMORE MS. "Killing of VRE Enterococcus faecalis by commensal strains: Evidence for evolution and accumulation of mobile elements in the absence of competition". Gut Microbes 7 (2016): 90-96.

14. Asadian M., et al. "Antimicrobial resistance pattern and genetic correlation in Enterococcus faecium isolated from healthy volunteers". Microbial Pathogenesis 92 (2016): 54-59. 
15. Hassan H and SHORMAN M. "Evaluation of the BD GeneOhm MRSA and VanR Assays as a Rapid Screening Tool for Detection of Methicillin-Resistant Staphylococcus aureus and Vancomycin-Resistant Enterococci in a Tertiary Hospital in Saudi Arabia". International Journal of Microbiology (2011): 861514.

16. Hegstad K., et al. "Performance of the EUCAST disk diffusion method, the CLSI agar screen method, and the Vitek 2 automated antimicrobial susceptibility testing system for detection of clinical isolates of Enterococci with low- and mediumlevel VanB-type vancomycin resistance: a multicenter study". Journal of Clinical Microbiology 52 (2014): 1582-1589.

17. Hashimoto Y., et al. "Dissemination and genetic analysis of the stealthy vanB gene clusters of Enterococcus faecium clinical isolates in Japan”. BMC Microbiology 18 (2018): 213.

18. Ozbak HA. "A novel high-resolution melting analysis approach for rapid detection of vancomycin-resistant enterococci". Annals of Saudi Medicine 38 (2018): 200-207.

19. Cheesbrough M. "Microbiological tests. District Laboratory Practice in Tropical Countries". Second Edition ed.: Cambridge University Press (2006).

\section{Assets from publication with us}

- Prompt Acknowledgement after receiving the article

- Thorough Double blinded peer review

- Rapid Publication

- Issue of Publication Certificate

- High visibility of your Published work

Website: https://www.actascientific.com/

Submit Article: https://www.actascientific.com/submission.php

Email us: editor@actascientific.com

Contact us: +919182824667 